\title{
Exploring Children's Everyday Journeys with User-Generated AR
}

\author{
Will Cash \\ Kate Howland \\ Department of Informatics \\ University of Sussex, UK

\section{Karina Rodriguez Echavarria}

\section{Myrsini Samaroudi} \\ Centre for Secure, Intelligent \\ and Usable Systems \\ University of Brighton, UK
}

\begin{abstract}
This paper describes the design of a tool to allow children to create their own Augmented Reality (AR) content as part of creative engagement with their local environment. We are exploring AR Maps (physical maps with augmented digital content) as a way of documenting children's experiences of their local area and encouraging their understanding and appreciation of objects and sites in their communities. We have piloted the approach with children in a local primary school, with positive feedback. However, children were not able to create the AR content themselves, and their physical artwork had to be scanned by researchers. In this paper we give an overview of the design context and describe the design and implementation of a tool to allow children to create their own 3D models for the AR environment by digitising their own artwork.
\end{abstract}

\section{Author Keywords}

Augmented reality; user-generated content; digital narratives; children

personal or classroom use is granted without fee provided that copies are not made or distributed for profit or commercial advantage and that copies bear this notice and the full citation on the first page. Copyrights for third-party components of this work must be honored. For all other uses, contact the Owner/Author.

IDC '20 Extended Abstracts, June 21-24, 2020, London, United Kingdom (C) 2020 Copyright is held by the owner/author(s).

ACM ISBN 978-1-4503-8020-1/20/06.

https://doi.org/10.1145/3397617.3397847

\section{CSS Concepts}

- Human-centered computing Human computer interaction $(\mathrm{HCI}) \sim$ Interaction paradigms Mixed / augmented reality 

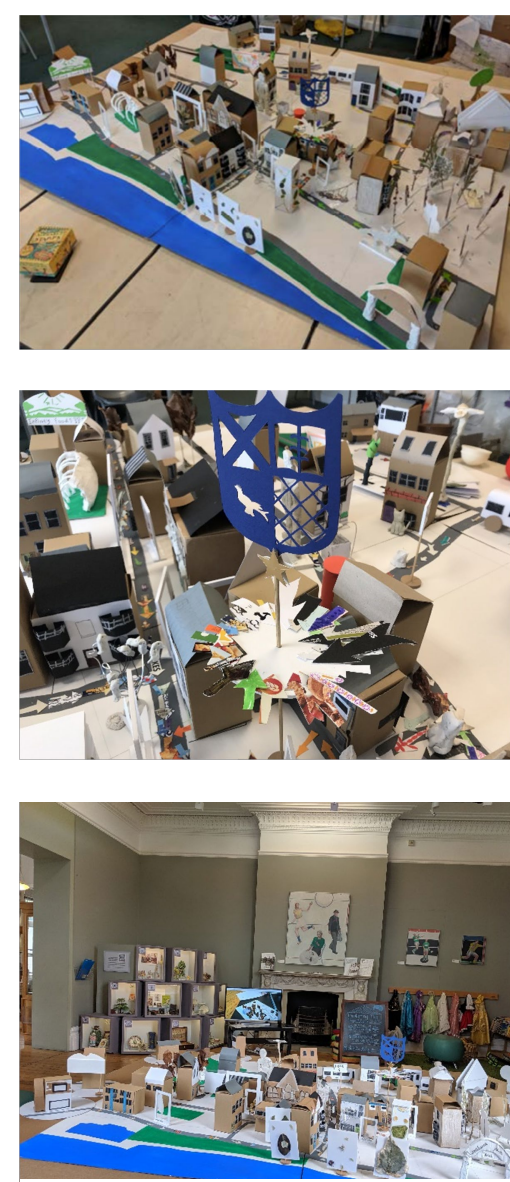

Figure 1: Physical models and map from pilot project

\section{Introduction}

Augmented Reality (AR) tools have been designed to support children's learning and play in varied contexts $[2,3,4,5,6]$. In some cases, AR has been used as a tool to support children's design and experimentation [4, $3]$, and other work has sought to involve children in the design of AR experiences for cultural and educational experiences [5, 6]. Our approach explores the potential for AR digitisation of map-based narratives produced by children as part of a cultural engagement project, to support the recording and sharing of their creative work [1]. In our pilot project in a primary school [1] we explored an approach using physical making and webbased AR as a way of encouraging children to connect with their cultural environment. Children's 3D artwork was digitized and placed in an AR environment by researchers, as there was no way for children to create the AR content themselves.

To allow children to create their own AR content in future iterations of this project, we have designed a tool that supports user-generated 3D AR content without the need for 3D graphics or AR expertise. There are commercially available tools that allow non-technical adult users to generate AR experiences, such as BlippAR's BlippBuilder [7] and Vuforia [8]. There are also some tools such as BlippAR's AR Butterfly [9], Disney's Colour and Play AR Colouring Book [10] and Crayola's Colour Alive AR Colouring Book [11] that allow children to customise an AR experience by colouring in a 2D page. However, there are no existing tools that allow children or non-technical users to create their own 3D content for AR.

The paper begins by outlining the design context by giving an overview of the pilot project [1]. We then describe the design process, before presenting the implemented tool, early conclusions and further work.

\section{Context: Pilot Project}

The pilot project [1] took place in collaboration with a local cultural organisation who deliver creative activities in schools to support children's wellbeing. An artist facilitated ten workshops at a local primary school using creative methods and psychogeography [12]

techniques to explore the everyday journeys children make from home to school. Thirty-two children aged 910 took part in the workshops over the course of one school term. The workshops explored a series of questions: how the children feel about travelling the same route five days a week, every week; how much they are aware of the physical environment around them on these journeys; where they look when they walk; and what they daydream about.

Children were encouraged to look at the streets where they walk more closely and from a different perspective, looking up and down where normally they would not, and studying the façade of the buildings in which they live. They also did additional research on the local area and its history, and were invited to see themselves as historians and creative observers of everyday life. With the artist's help, they developed narratives which incorporated their own journeys and elements from the geographical landscape, using a mixture of physical, visual and textual material. Each child decorated a box which represented their house, as shown in Figure 1, and used this as a container for the materials telling their personal narrative. Some children wrote textual content with stories of people, objects, sites and events they had researched. Finally, all elements were organised according to their 

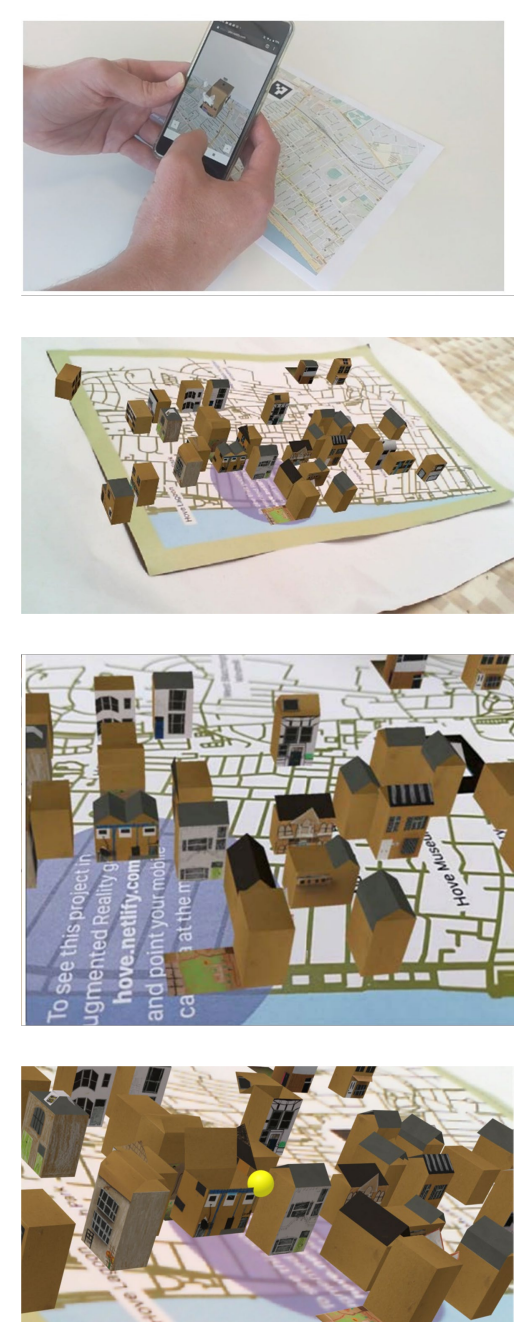

Figure 2: Viewing AR Maps geographical location on a physical map, showing the collective journeys made by the children converging at the shared community of school (see Figure 1).

The outcomes of psychogeography and arts-based community engagement are typically not kept after such workshops have finished. Our pilot project explored the potential for the digitisation of the narratives produced by children to support i) their documentation and preservation; and ii) the re-telling of these narratives to others. The audiences for this could include family and friends, as well as stakeholders who want to understand which elements of the cultural landscape are significant to children.

In the pilot project, the process of digitisation was completed offline after the workshops had finished. The models were photographed and the photos turned into texture maps and applied to 3D models using the 3D modelling software, Blender. The 3D models were saved in a gITF file format and incorporated into a webbased AR experience. The experience is viewed in a web browser, with the platform implemented using HTML/CSS/JavaScript and the A-Frame framework [13] and AR.js libraries [14]. The outcome was an AR Map of the local area that worked with a printed map that included a custom fiducial marker based on the school's logo. To view the experience, the user scans the

fiducial marker to produce the whole map of AR models [1], as shown in Figure 2.

At the end of the project, a celebration of the artwork and the children's journey took place at a local museum, and children were invited to bring their families and friends to view the artwork and the digital experience. Children were keen to test the printed map on their phones and look for their house and their stories, and the digital experience generated positive feedback from children and families [1].

There is interest from schools and local collaborators in running further iterations of the project. However, the current digitisation process is problematic because children are not able to create the AR content themselves. This means they are detached from the process and that the approach does not scale well.

\section{Design}

The requirements for the tool were established through a series of meetings with key stakeholders from the pilot project, including representatives from the cultural organisation, researchers and the developers of the AR environment. We identified the need for a tool that allows children to create their own 3D AR content, under the supervision of a teacher or parent, focusing on the creation of the building models that are central to the experience. To support use in as many school and home settings as possible, it was determined that the tool should be a web-application that could run on mobile devices, tablets and desktop machines. A fast and simple digitisation process was identified as being more important than achieving a very high quality final model. For this reason we determined that single photos of each face of the buildings would be captured, converted to textures and applied to 3D model templates.

To allow flexibility for users, the tool needed to support photos either being taken with the device running the application or the uploading of photos that had been previously captured on another device, e.g. flat-bed scanner. The artists running the workshops used three different sizes of foldable boxes as the foundation of the 


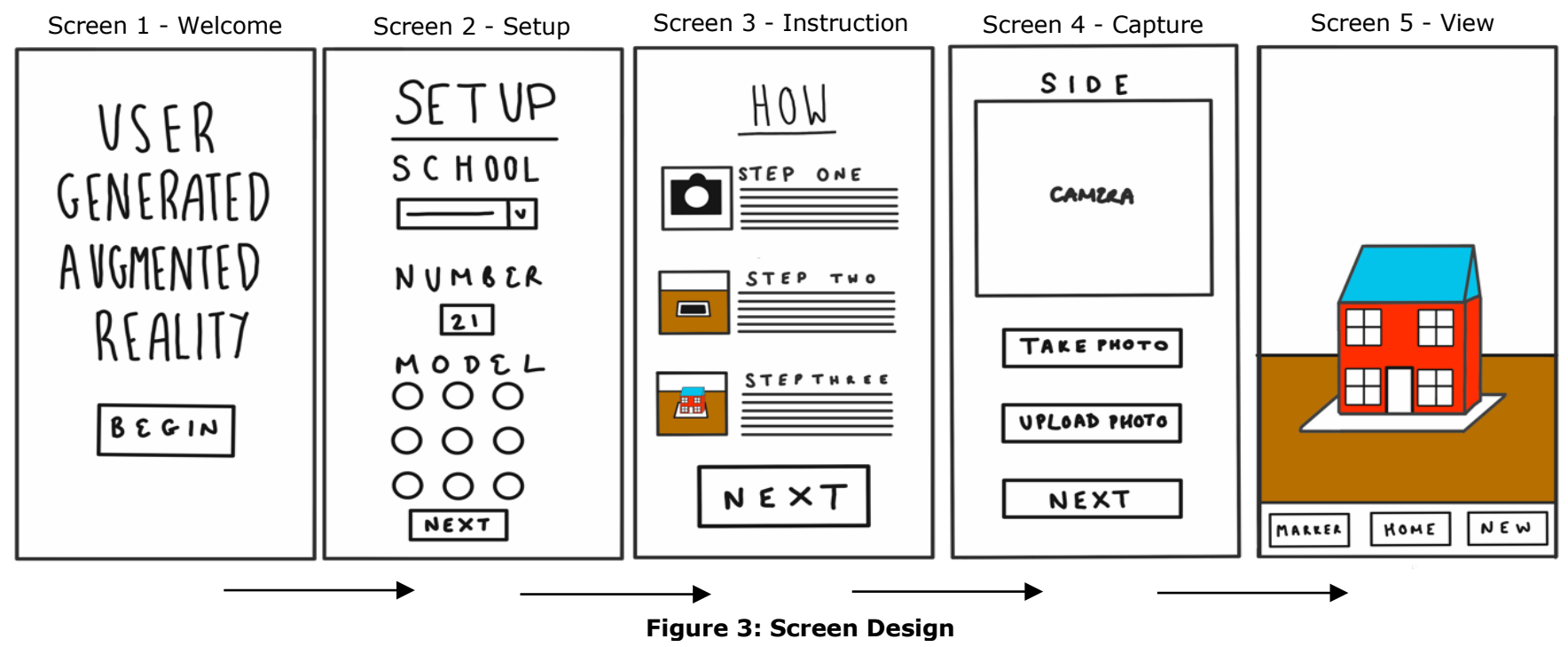

houses, and these could each be placed in three different configurations, giving nine different model templates the tool needed to support. To make the task achievable by 9-10 year olds, we designed a very simple workflow in which one face at a time is added to the model.

Figure 3 shows the initial mock-up for the design. The application starts with a title screen (Screen 1) comprising the title of the application and a button to launch the model creation process. Upon launching, the user is taken to the setup screen (Screen 2), where they choose their school from a dropdown menu, enter a model ID (assigned to them by their teacher) and choose the configuration of their model (including the type of roof). Following this, the user is taken to an instructional screen (Screen 3) that explains the content capture process and how the user views their model at the end.

Screen 4 represents the Content Capture stage and consists of a camera viewport and three buttons: 'Take Photo', 'Upload Photo' and 'Next'. The camera viewport is used to capture the content, and each of the nine configurations has separate pages viewports constrained to the size of each side of the cardboard model. By constraining the camera's aspect ratio to the sizes of the sides of the model, the content captured is a $1: 1$ match with little room for error. The only errors that could be introduced would be the choice of the wrong configuration of the model and the positioning and taking of the photos. The two buttons 'Take Photo' and 'Upload Photo' are necessary for the content capture because the photo will require a trigger and the content (regardless of whether it has been captured in the application or via another device), will need to be uploaded to the project file-space. Incorporating these buttons meets the functional requirements of allowing the user to capture and upload their content via the application. 

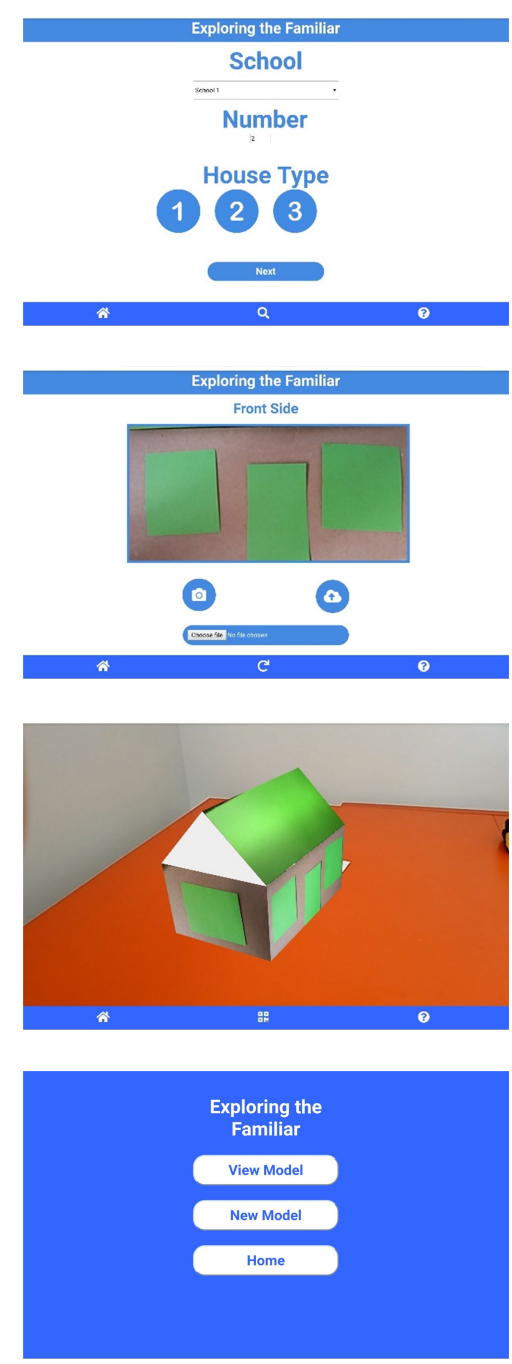

Figure 4: UGAR Web Application (Screens from top to bottom: setup, capture, view, options)
Once the user has been through all states of the Content Capture stage and has content for all sides of the model, they are taken to the view model screen (Screen 5). In this state, by scanning the project's dedicated fiducial marker, the user will be able to see their virtual model. Additional functionalities included in the sketch include the ability to download the marker (should the user not have access to one) and the ability to create a new model. The ability to create a new model allows for repeating the process with a new model.

\section{User Generated Augmented Reality (UGAR)}

The User Generated Augmented reality (UGAR) web application was implemented using HTML, CSS and JavaScript. To ensure compatibility with the existing AR platform, we used the A-Frame framework, AR.js libraries and a fiducial marker-based approach.

After entering their school details and model ID number, the user can capture, download and upload their content. As described above, the application contains viewports for each possible configuration of the models. It captures content in the order: front, left, right, back and top/roof elements, with instructions directing the user in what content to capture when. The viewports and workflow ensure the content is captured and applied to each face correctly, minimising the possible errors that could be made by anyone unfamiliar with the process of creating 3D models.

Upon capturing the content, the next step involves transferring and storing the user's content so it can be used to create a distinct model. UGAR achieves this with each user having a dedicated folder, which stores their content and model. The application uses PHP to transfer and store each piece of content in the user's dedicated folder. The application is able to manage producing multiple models at once because the models are stored separately. The images stored in the model folder are renamed to match the corresponding face of the cardboard model. These definitions are used in the model file to make a generic model template that is copied and used to produce multiple unique models.

The final stage is applying the user's content to the model, since the user captures each face's content individually. By default, A-Frame applies the same texture to each face of an object repeatedly, as opposed to matching the texture face to face. This issue changed the original process for UGAR, which would have involved stitching the images together to create a cubemap texture. Instead, an external

component (developed for A-Frame) was identified that allows face to face applications of texture. Through the use of this component, the application is able to apply unique textures to each face of the model.

\section{Conclusion and Further Work}

We have described the design and implementation of UGAR, a tool that makes it possible for children (under the supervision of a teacher or parent) to create their own 3D models for use on an AR Map. To simplify the complex process of creating a 3D model with a custom texture create from photographs, we have adopted a standardised workflow that is constrained to the needs of the project context. It supports the creation of models in nine different configurations, in line with the templates used in the everyday journeys workshops.

The next step is to run user testing sessions with children and teachers to identify any usability and user experience issues to be tackled before deployment in 
schools in the next iteration of the project. We will examine how challenging it is for children to capture and upload photos of their models using the application, including identifying the correct configuration of their model, and find ways to simplify the process if necessary. Following this, we will run a more extensive evaluation to investigate how allowing children to generate 3D AR models from their artwork changes the experience, and further explore the opportunities created.

\section{Acknowledgements}

We thank all the children and teachers who took part in the pilot project and the artists and policy makers at Future Creators. We also thank Mnemoscene, who developed the AR environment for the pilot project.

\section{References}

[1] H. T. Zimmerman, S. M. Land, M. R. Mohney, G. W. Choi, C. Maggiore, S. H. Kim, Y. J. Jung and J. Dudek, "Using augmented reality to support observations about trees during summer camp," in Proceedings of Interaction Design and Children (IDC '15), 2015.

[2] S. Kang, L. Norooz, E. Bonsignore, V. Byrne, T. Clegg and J. E. Froehlich, "PrototypAR:

Prototyping and Simulating Complex Systems with Paper Craft and Augmented Reality," in Proceedings of Interaction Design and Children (IDC '19), 2019.

[3] S. Oberhuber, T. Kothe, S. Schneegass and F. Alt, "Augmented Games: Exploring Design Opportunities in AR Settings With Children," in Proceedings of Interaction Design and Children (IDC '17), 2017.

[4] H. Alhumaidan, K. P. Y. Lo and A. Selby, "Codesigning with children a collaborative augmented reality book based on a primary school textbook," International Journal of ChildComputer Interaction, vol. 15, pp. 24-36, 2018.

[5] T. Schofield, D. T. Pisanty, G. Arrigoni, K. Reynolds and R. Pattinson, "Magical Realism and Augmented Reality: Designing Apps with Children in a Cultural Institution," in Proceedings of Designing Interactive Systems (DIS '19), 2019.

[6] K. R. Echavarria, L. Dibble, A. Bracco, E. Silverton and S. Dixon, "Augmented Reality (AR) Maps for Experiencing Creative Narratives of Cultural Heritage," in EUROGRAPHICS Workshop on Graphics and Cultural Heritage (2019), 2019.

[7] BlippAR, "BlippAR BlippBuilder," BlippAR, [Online]. Available: https://www.blippar.com/build-ar.

[8] Vuforia, "Vuforia Developer Portal," [Online]. Available: https://developer.vuforia.com/.

[9] BlippAR, "Butterfly," [Online]. Available: https://www.blippar.com/work/butterfly.

[10] Disney, "Disney Colour and Play Books by Bendon," [Online]. Available: https://www.colorandplaybooks.com/.

[11] Crayola, "Colour Alive," [Online]. Available: http://www.crayola.co.uk/splash/product/colouralive.aspx

[12] M. Coverley, Psychogeography, Harpenden Pocket Essentials, 2006.

[13] A-Frame, "A-Frame - Introduction," 2020. [Online]. Available:

https://aframe.io/docs/0.9.0/introduction/.

[14] J. Etienne, "AR.js - Augmented Reality for the Web," [Online]. Available: https://jeromeetienne.github.io/AR.js/. [Accessed 1 October 2019].

[15] Khronos Group, "glTF Overview," [Online]. Available: https://www.khronos.org/gltf/. 